\title{
The Efficacy and Safety of GCWB104 (Flos Lonicera Extract) in Functional Dyspepsia: A Single-Center, Randomized, Double-Blind, Placebo-Controlled Study
}

\author{
Yonghoon $\mathrm{Choi}^{1}$, Nayoung Kim ${ }^{1,2}$, Gi Tark Noh ${ }^{1}$, Ju Yup Lee ${ }^{3}$, and Dong Ho Lee ${ }^{1,2}$ \\ ${ }^{1}$ Department of Internal Medicine, Seoul National University Bundang Hospital, Seongnam, ${ }^{2}$ Department of Internal Medicine and Liver \\ Research Institute, Seoul National University College of Medicine, Seoul, and ${ }^{3}$ Department of Internal Medicine, Keimyung University School \\ of Medicine, Daegu, Korea
}

Background/Aims: The Flos Lonicera extract GCWB104 has been shown to have significant protective effects against gastritis and gastric ulcers in vivo. The aim of this study was to investigate the efficacy and safety of GCWB104 in subjects with functional dyspepsia (FD). Methods: In this singlecenter, double-blind, randomized clinical trial, 92 subjects diagnosed with FD using the Rome III criteria were allocated to either the test group (300 mg of GCWB104, containing 125 $\mathrm{mg}$ of Flos Lonicera extract, twice daily) or the placebo group (300 mg placebo, twice daily). The total score improvement on the Gastrointestinal Symptom Rating Scale (GSRS) for individual symptoms, changes in antioxidant levels, changes in dyspepsia-related quality of life according to the Nepean Dyspepsia Index (NDI), and adverse effects were compared before and after 8 weeks of treatment. Results: The differences in total GSRS scores and score improvements after 8 weeks of treatment were significant between the GCWB104 and control groups $(p=0.0452$ and $p=0.0486$, respectively). Thirteen of 15 individual symptoms on the GSRS improved in the GCWB104 group, while six symptoms improved in the control group. In addition, statistically significant changes in rumbling, loose stool, and stool urgency were observed in the GCWB104 group. Blood 8-hydroxy-2'-deoxyguanosine (8OHdG) levels, known as antioxidants, showed significant reductions after 8 weeks of administration of GCWB104. There were no adverse events related to treatment with GCWB104. Conclusions: GCWB104 safely contributed to improvements in mild to moderate FD and irritable bowel syndrome symptoms. Antioxidant effects of GCWB104 were also suggested (Clinicaltrials.gov number NCT04008901). (Gut Liver 2020;14:67-78)
Key Words: Lonicera; Dyspepsia; Antioxidant

\section{INTRODUCTION}

Dyspepsia is a common symptom that accounts for up to $5 \%$ of patients who visit their primary health care provider, and functional dyspepsia (FD) accounts for 60\% to $80 \%$ of all patients with dyspepsia. ${ }^{1,2}$ FD is a common syndrome in which chronic, repetitive gastrointestinal (GI) symptoms occur predominantly in the upper GI tract, with no apparent underlying disease. Rome III criteria categorize FD as two subtypes: postprandial distress syndrome and epigastric pain syndrome, according to symptoms of postprandial fullness, early satiety, stomach pain, and epigastric soreness. In a recent nationwide study of FD following the Rome III criteria, the prevalence of FD was $10.3 \%$ in Korea, with prevalence of a postprandial distress syndrome subtype of 7.3\%, prevalence of an epigastric pain syndrome subtype of 5.5\%, and approximately 25\% of FD patients categorized with overlap syndrome. ${ }^{3}$

The pathophysiological mechanisms of FD may be multifactorial and remain to be fully elucidated, although widely recognized mechanisms are gastroduodenal dysmotility, gastroduodenal acid exposure, visceral hypersensitivity, autonomic/ central nervous system dysfunction, Helicobacter pylori infection, and psychosomatic factors. ${ }^{4,5}$ Standard management of FD has not yet been established, and satisfactory pharmacotherapy is also unavailable. Anti-secretory drugs, such as proton pump inhibitors, prokinetics, histamine receptor antagonists, and antidepressants, have been evaluated extensively for FD therapy despite little evidence of efficacy. ${ }^{6}$ Natural product extracts have

Correspondence to: Nayoung Kim

Department of Internal Medicine, Seoul National University Bundang Hospital, 82 Gumi-ro 173beon-gil, Bundang-gu, Seongnam 13620, Korea

Tel: +82-31-787-7008, Fax: +82-31-787-4051, E-mail: nakim49@snu.ac.kr

Received on August 13, 2019. Revised on November 10, 2019. Accepted on November 26, 2019.

pISSN 1976-2283 eISSN 2005-1212 https://doi.org/10.5009/gnl19283

() This is an Open Access article distributed under the terms of the Creative Commons Attribution Non-Commercial License (http://creativecommons.org/licenses/by-nc/4.0) which permits unrestricted non-commercial use, distribution, and reproduction in any medium, provided the original work is properly cited. 
recently attracted attention, since they have comprehensive treatment effects and are relatively safe.

GCWB104 is a newly formulated extract obtained from Flos Lonicera, a dried bud of the genus Lonicera japonica thunberg belonging to the family Caprifoliaceae. Flos Lonicera extracts are known to have been used as a crude medicine for abdominal symptoms in East Asia since the Ming Dynasty of China. The major component of Flos Lonicera is chlorogenic acid, which has been shown to have significant anticarcinogenic, antiinflammatory, antioxidant, and analgesic effects in an animal gastritis, gastric ulcer model. In addition, Flos Lonicera extracts showed excellent gastric motor improvement in the gastric emitting and GI transmission model. ${ }^{7-9}$ Therefore, ingestion of GCWB104 is expected to protect and improve the function of the stomach. In addition, in reflux esophagitis model, which is related to FD, the Flos Lonicera extract improved the esophageal lesion score and histopathologic changes. ${ }^{10,11}$ From this background, the aim of this study was to investigate the efficacy of GCWB104 on the improvement of mild to moderate FD symptoms compared with placebo.

\section{MATERIALS AND METHODS}

\section{Study participants}

This trial recruited a total of 92 people from February 2017 to July 2018. Male and female subjects $>19$ years of age who had mild to moderate FD were eligible. They had baseline endoscopic screening, and subjects without active lesions (peptic ulcer disease or gastric erosions) were enrolled. Written informed consent was obtained from all participants.

Exclusion criteria included: allergic history to natural substances and ingredients, need for medical therapy for reflux esophagitis (above LA-A), acute gastritis or gastric ulcers on esophagogastroduodenoscopy, inability to undergo endoscopy, history of esophageal or gastric surgery that could affect GI motility, continuation of steroids, nonsteroidal anti-inflammatory drugs, or other daily medications that can cause ulcers, severe liver disease (above 2.5 times the upper limit of normal for aspartate aminotransferase, alanine aminotransferase, gamma glutamyl transferase), chronic kidney disease, renal failure, severe cardiovascular or pulmonary diseases, unregulated diabetes, cerebrovascular diseases, need for surgery during the test period, administration of proton pump inhibitors, H2-receptor antagonist, gastroesophageal reflux disease treatment medication within 2 weeks of endoscopic examination or 1 week before the first visit, pregnant or breastfeeding females, planning conception or disagreement with proper contraception methods, and participation in other human trial within 4 weeks before the trial. The number of remnant products was counted in visit 3 and visit 4, and the lower limit of trial food compliance was $80 \%$. Subjects with compliance less than $80 \%$ were considered as non-compliance group and excluded from the study.

\section{Study design}

This study was a single-center, double-blind, randomized, placebo-controlled clinical trial conducted in South Korea. The study protocol was approved by the Seoul National University Bundang Hospital Institutional Review Board (IRB number: BST104-01), and this trial was registered with Clinicaltrials. gov (number: NCT04008901) and the Korean Clinical Research Information Service (number: KCT0004085). FD was defined by Rome III criteria as person with one or more symptoms of epigastric pain or soreness, postprandial fullness, or early satiety for the past 3 months with symptom onset at least 6 months prior to diagnosis; no evidence of structural disease that was likely to explain symptoms; and no organic lesion detected by endoscopic examination. Screening tests were conducted on each person before 1 week of the scheduled first administration, and exclusion of those who were not suitable was based on the inclusion and exclusion criteria. The registered subjects were given an oral dose of GCWB104 or placebo for 8 weeks after random assignment, and the efficacy and safety were assessed by visiting the institution at 4 weeks and 8 weeks after administration.

\section{Number of target subjects and basis of calculation}

The number of subjects was calculated as follows: referring to the prior study about the efficacy of Abexol using GSRS score, ${ }^{12}$ the study showed that the average change of GSRS in placebo group was -2.7 after 8 weeks, and the average change of GSRS in Abexol group was -6.1. The difference of change of GSRS between the trial group and the placebo group was -3.4. The effect of GCWB104 was estimated to -2.5 , about $73.5 \%$ of Abexol, and the standard deviation was estimated to 3.4, the maximum standard deviation in the reference text. And the number of participants in each group required for this study was calculated to 30, referring to the central limit theorem, which states that even if the population does not follow a normal distribution, if the sample size is 30 or higher, the distribution of the sample mean is approximated to the normal distribution. In addition, we assumed the dropout rate for each group to be $34 \%$. A total of 92 people would be needed, considering that the efficacy of GCWB104 was assumed to be 73\% that of Abexol with an approximately 34\% elimination rate; in addition, a type I error of 5\% and type II error of 20\% were assumed, referring to the previous clinical trial focusing on the efficacy of Abexol in subjects with FD. ${ }^{12}$

\section{Methods of efficacy measurement}

A week before administering the drug, collection of the consent form, checking of the vital signs, pregnancy tests, and blood and urine tests were performed. After random assignment, 300 mg of GCWB104 (including 125 mg of Flos Lonicera 
extract) or placebo was administered per oral twice daily, after a meal, for a total of 8 weeks. At 4 and 8 weeks after administration, vital signs and compliance were checked, and symptom evaluation was performed using a Gastrointestinal Symptom Rating Scale (GSRS) questionnaire. At 8 weeks after administration, physical examination and urea breath test for $H$. pylori were performed. Random assignment was performed using the permuted block randomized method, and the trial was designed as double-blind to minimize bias. During the trial period, medications that could affect clinical trials were prohibited, and the individual history of drug dosing was checked through a questionnaire at each visit.

The primary efficacy was defined as the total GSRS score improvement after 8 weeks of administration compared to baseline. The GSRS is a symptom-specific instrument of 15 items combined into five symptom clusters depicting reflux, abdominal pain, indigestion, diarrhea, and constipation. It has a 4-point graded Likert-type scale where 1 represents the absence of troublesome symptoms and 4 represents very troublesome symptoms, and the reliability and validity of the GSRS are well documented..$^{13}$ In addition, the second efficacy was defined as the improvement rate in GSRS score (total GSRS [\%], the percentile improvement rate, converted value of each of the 15 items to \% together), the improvement of individual symptoms, the change of serum antioxidant biomarker levels, and the improvement of dyspepsia-related quality of life using the Nepean Dyspepsia Index (NDI) after 8 weeks of administration compared to baseline. The NDI questionnaire consisted of five categories and 25 questions of GI symptoms, with each question rated on a 5-point Likert scale. And an additional analysis was done based on $90 \%$ compliance, to check the relationship between higher compliance and signiticant efficacy. Totally 59 subjects showed compliance over 90\%, 33 in the GCWB104 group and 26 in the control group.

\section{Measurement of antioxidative markers}

Blood samples from a forearm vein were drawn with the subjects in a fasting state (i.e., fasting for at least 8 hours prior). The serum samples were immediately separated by centrifugation at $3000 \mathrm{~g}$ for 10 minutes and were stored at $-80^{\circ} \mathrm{C}$ for further analysis, based on a previous study on antioxidative biomarkers. ${ }^{14}$ Commercially available assay kits were used to perform the assay for 8-hydroxy-2'-deoxyguanosine (8-OHdG), thiobarbituric acid reactive substances, and high sensitivity C-reactive protein (Cayman Chemical Co., Ann Arbor, MI, USA). All analyses were performed at the same laboratory (GC LabCell, Yongin, Korea).

\section{Safety issues}

The adverse events, adverse drug reactions, and significant adverse events that occurred after drug exposure were investigated, and the rate differences of adverse events, abnormal drug reactions, and significant adverse events were compared and analyzed among treatment groups. Changes in vital signs, and laboratory tests were also compared and analyzed after 4 weeks and 8 weeks of administration.

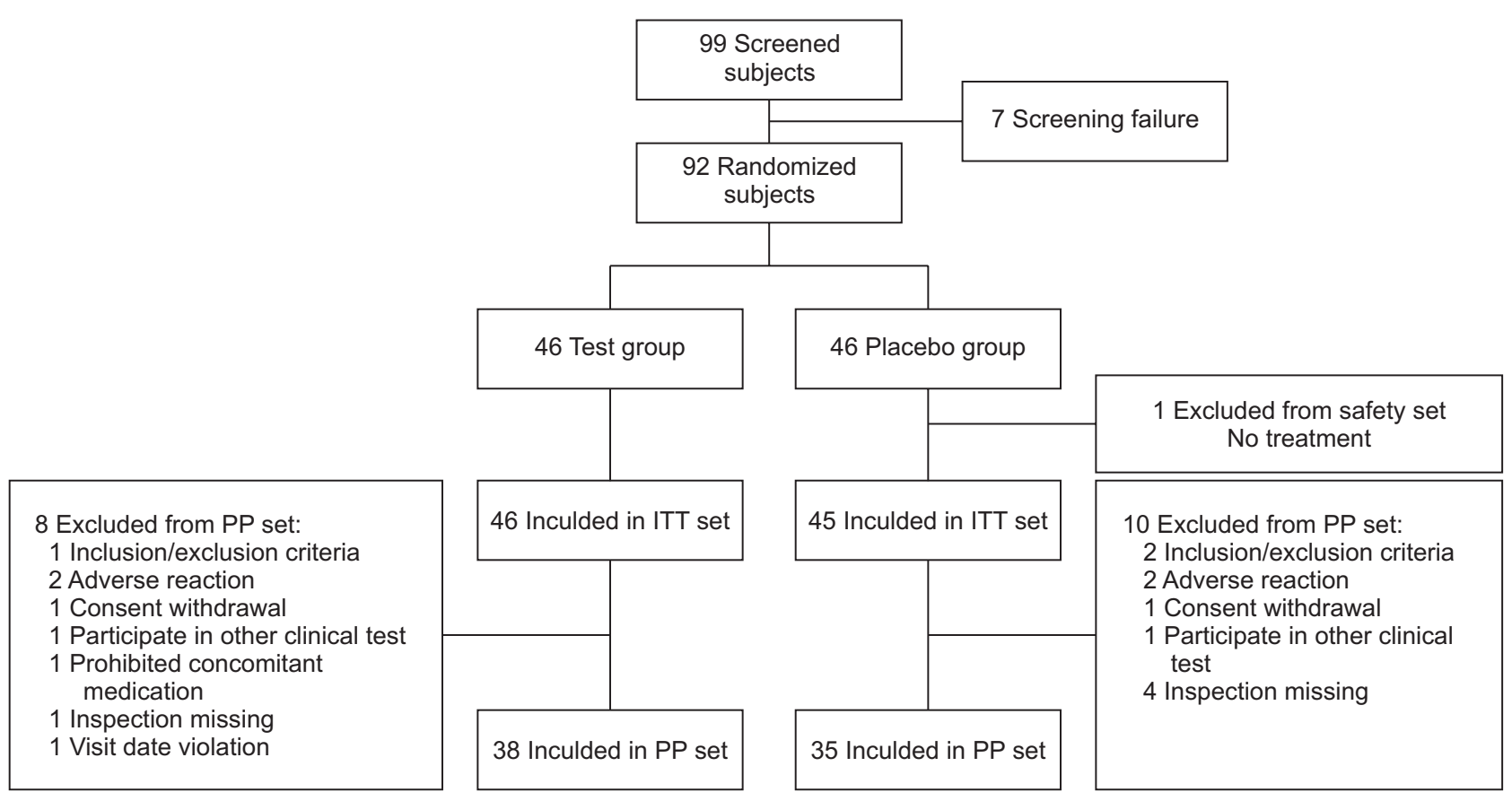

Fig. 1. Flowchart showing enrolled subjects and dropouts from the study. Adverse reaction definitely related to study drug: rash ( $\mathrm{n}=1$ ). Adverse reactions not related to study drug: musculoskeletal pain $(n=1)$, dyspepsia $(n=1)$ and nasal obstruction $(n=1)$.

ITT, intention-to-treat; PP, per protocol. 


\section{Statistical analysis}

The data obtained from the subjects of this trial were analyzed in three main forms: safety, full analysis (FA), and per protocol (PP). The safety group was defined as being randomly assigned to this human trial and took trial products at least once. The FA group was defined as having data of the efficacy evaluation collected more than once after administration of the trial product. The PP group was defined as the FA group who completed this human testing without significant plan violations, and in this case, only the following randomly assigned

Table 1. Baseline Characteristics of Study Participants

\begin{tabular}{|c|c|c|c|c|}
\hline Variable & $\begin{array}{c}\text { GCWB104 } \\
(\mathrm{n}=38)\end{array}$ & $\begin{array}{l}\text { Control } \\
(n=35)\end{array}$ & $\begin{array}{l}\text { Total } \\
(n=73)\end{array}$ & p-value ${ }^{*}$ \\
\hline Sex & & & & 0.1065 \\
\hline Male & $10(26.32)$ & $4(45.95)$ & $14(19.18)$ & \\
\hline Female & 28 (73.68) & $31(54.05)$ & $59(80.82)$ & \\
\hline Age, yr & $50.87 \pm 11.20$ & $50.43 \pm 12.40$ & $50.66 \pm 11.71$ & 0.8901 \\
\hline Height, cm & $161.98 \pm 9.26$ & $159.95 \pm 5.45$ & $161.01 \pm 7.69$ & 0.5801 \\
\hline Weight, kg & $61.04 \pm 11.16$ & $55.86 \pm 7.44$ & $58.56 \pm 9.84$ & $0.0421^{*}$ \\
\hline Drinking & & & & 0.3357 \\
\hline Current & $16(42.11)$ & $11(31.43)$ & 27 (36.99) & \\
\hline Ex & $1(2.63)$ & 0 & $1(1.37)$ & \\
\hline Never & $21(55.26)$ & $24(68.57)$ & 45 (61.64) & \\
\hline Current medical history $^{\dagger}$ & & & & - \\
\hline Hypothyroidism & $1(2.63)$ & 0 & $1(1.37)$ & \\
\hline Chronic gastritis & $18(47.37)$ & $8(22.86)$ & $26(35.62)$ & \\
\hline Diverticulum & $1(2.63)$ & 0 & $1(1.37)$ & \\
\hline Duodenal ulcer & $3(7.89)$ & 0 & $3(4.11)$ & \\
\hline Gastric xanthoma & $1(2.63)$ & 0 & $1(1.37)$ & \\
\hline Gastritis & $1(2.63)$ & $1(2.86)$ & $2(2.74)$ & \\
\hline Erosive gastritis & $4(10.53)$ & $9(25.71)$ & $13(17.81)$ & \\
\hline Hiatus hernia & $2(5.26)$ & 0 & $2(2.74)$ & \\
\hline Intestinal metaplasia & $4(10.53)$ & $1(2.86)$ & $5(6.85)$ & \\
\hline Gastric mucosal hypertrophy & 0 & $1(2.86)$ & $1(1.37)$ & \\
\hline Gastric polyps & 0 & $1(2.86)$ & $1(1.37)$ & \\
\hline Gastroesophageal reflux disease & 0 & $1(2.86)$ & $1(1.37)$ & \\
\hline Drug hypersensitivity & $1(2.63)$ & 0 & $1(1.37)$ & \\
\hline Food allergy & 0 & $1(2.86)$ & $1(1.37)$ & \\
\hline Diabetes mellitus & $1(2.63)$ & $1(2.86)$ & $2(2.74)$ & \\
\hline Hyperlipidaemia & $3(7.89)$ & $6(17.14)$ & $9(12.33)$ & \\
\hline Osteoporosis & $1(2.63)$ & $2(5.71)$ & $3(4.11)$ & \\
\hline Back pain & 0 & $1(2.86)$ & $1(1.37)$ & \\
\hline Skin papilloma & $1(2.63)$ & 0 & $1(1.37)$ & \\
\hline Gastrointestinal submucosal tumor & 0 & $2(5.71)$ & $2(2.74)$ & \\
\hline Hypertension & $2(5.26)$ & $4(11.43)$ & $6(8.22)$ & \\
\hline Staphyloma & 0 & $1(2.86)$ & $1(1.37)$ & \\
\hline Headache & 0 & $1(2.86)$ & $1(1.37)$ & \\
\hline Benign prostatic hyperplasia & 0 & $1(2.86)$ & $1(1.37)$ & \\
\hline Cystitis & $1(2.63)$ & 0 & $1(1.37)$ & \\
\hline
\end{tabular}

Data are presented as number (\%) or mean \pm SD.

${ }^{*} \mathrm{p}<0.05$; $\mathrm{p}$-value indicates statistical significance for continuous variables tested with the Mann-Whitney U-test; categorical variables tested with Pearson chi-square test; smoking tested with Fisher exact test. ${ }^{\dagger}$ Overlap of multiple diseases in the same patient included. 
targets were excluded from the PP analysis. The efficacy data were analyzed for both the FA and PP groups but mainly for the PP group. The evaluation of safety data was conducted on the safety group, and the demographic and baseline characteristics were analyzed for the PP group. As descriptive statistics, the number, mean, standard deviation, median, minimum, and maximum values were presented for continuous variables, and the number and percentage of the test subjects and frequency, if needed, were presented for categorical variables. The significance of the difference between treatment groups at each visit time was analyzed using the independent two-sample t-test or Mann-Whitney U-test for continuous variables and Pearson chisquare test or Fisher exact test for categorical data. The significance of the difference between treatment groups between the two measurement points was analyzed using the independent two-sample test or Mann-Whitney U-test for continuity vari-

Table 2. Total GSRS Scores of Both Groups at Baseline, the 4th, and the 8th Week

\begin{tabular}{|c|c|c|c|c|}
\hline Variable & Treatment & Baseline & 4 Weeks & 8 Weeks \\
\hline \multirow[t]{2}{*}{ Abdominal pain } & Placebo & $1.29 \pm 0.52$ & $1.14 \pm 0.36$ & $1.11 \pm 0.32$ \\
\hline & GCWB104 & $1.50 \pm 0.76$ & $1.18 \pm 0.39 *$ & $1.08 \pm 0.27^{\dagger}$ \\
\hline \multirow[t]{2}{*}{ Acidity \& heartburn } & Placebo & $1.63 \pm 0.73$ & $1.20 \pm 0.41^{\dagger}$ & $1.17 \pm 0.45^{\dagger}$ \\
\hline & GCWB104 & $1.87 \pm 0.84$ & $1.37 \pm 0.54^{\ddagger}$ & $1.13 \pm 0.34^{\S}$ \\
\hline \multirow[t]{2}{*}{ Acid reflux } & Placebo & $1.49 \pm 0.66$ & $1.20 \pm 0.41^{*}$ & $1.26 \pm 0.44$ \\
\hline & GCWB104 & $1.68 \pm 0.77$ & $1.53 \pm 0.65$ & $1.24 \pm 0.43^{\dagger}$ \\
\hline \multirow[t]{2}{*}{ Sensation of stomach emptiness } & Placebo & $1.49 \pm 0.66$ & $1.14 \pm 0.36^{*}$ & $1.17 \pm 0.45^{*}$ \\
\hline & GCWB104 & $1.63 \pm 0.79$ & $1.47 \pm 0.60$ & $1.08 \pm 0.27^{\ddagger}$ \\
\hline \multirow[t]{2}{*}{ Nausea \& vomiting } & Placebo & $1.31 \pm 0.53$ & $1.17 \pm 0.38$ & $1.09 \pm 0.28^{*}$ \\
\hline & GCWB104 & $1.42 \pm 0.60$ & $1.32 \pm 0.53$ & $1.05 \pm 0.23^{\ddagger}$ \\
\hline \multirow[t]{2}{*}{ Abdominal noises } & Placebo & $1.46 \pm 0.70$ & $1.31 \pm 0.53$ & $1.34 \pm 0.54$ \\
\hline & GCWB104 & $1.66 \pm 0.78$ & $1.29 \pm 0.46^{\dagger}$ & $1.08 \pm 0.27^{\S}$ \\
\hline \multirow[t]{2}{*}{ Abdominal bloating } & Placebo & $1.77 \pm 0.60$ & $1.46 \pm 0.56^{*}$ & $1.40 \pm 0.50^{\dagger}$ \\
\hline & GCWB104 & $2.03 \pm 0.88$ & $1.61 \pm 0.64^{\dagger}$ & $1.32 \pm 0.47^{\S}$ \\
\hline \multirow[t]{2}{*}{ Eructation } & Placebo & $1.54 \pm 0.56$ & $1.34 \pm 0.48$ & $1.29 \pm 0.46^{*}$ \\
\hline & GCWB104 & $1.79 \pm 0.81$ & $1.50 \pm 0.51^{*}$ & $1.24 \pm 0.43^{\S}$ \\
\hline \multirow[t]{2}{*}{ Flatulence } & Placebo & $1.77 \pm 0.55$ & $1.43 \pm 0.56^{*}$ & $1.26 \pm 0.44^{\ddagger}$ \\
\hline & GCWB104 & $1.92 \pm 0.67$ & $1.53 \pm 0.56^{\dagger}$ & $1.53 \pm 0.68^{\dagger}$ \\
\hline \multirow[t]{2}{*}{ Slow intestinal transit } & Placebo & $1.34 \pm 0.59$ & $1.20 \pm 0.47$ & $1.20 \pm 0.58$ \\
\hline & GCWB104 & $1.39 \pm 0.64$ & $1.21 \pm 0.47^{*}$ & $1.16 \pm 0.37^{\dagger}$ \\
\hline \multirow[t]{2}{*}{ Accelerated intestinal transit } & Placebo & $1.11 \pm 0.32$ & $1.09 \pm 0.28$ & $1.06 \pm 0.24$ \\
\hline & GCWB104 & $1.24 \pm 0.43$ & $1.24 \pm 1.47$ & $1.11 \pm 0.31$ \\
\hline \multirow[t]{2}{*}{ Soft feces } & Placebo & $1.23 \pm 0.55$ & $1.31 \pm 0.47$ & $1.20 \pm 0.41$ \\
\hline & GCWB104 & $1.58 \pm 0.60$ & $1.47 \pm 0.51$ & $1.26 \pm 0.45^{*}$ \\
\hline \multirow[t]{2}{*}{ Hard feces } & Placebo & $1.71 \pm 1.05$ & $1.43 \pm 0.70$ & $1.37 \pm 0.81$ \\
\hline & GCWB104 & $1.34 \pm 0.67$ & $1.50 \pm 0.86$ & $1.29 \pm 0.52$ \\
\hline \multirow[t]{2}{*}{ Urgency for defecation } & Placebo & $1.17 \pm 0.38$ & $1.20 \pm 0.41$ & $1.23 \pm 0.49$ \\
\hline & GCWB104 & $1.45 \pm 0.65$ & $1.29 \pm 0.52$ & $1.13 \pm 0.41^{*}$ \\
\hline \multirow[t]{2}{*}{ Sensation of incomplete emptiness } & Placebo & $1.69 \pm 0.72$ & $1.51 \pm 0.61$ & $1.51 \pm 0.61$ \\
\hline & GCWB104 & $1.71 \pm 0.61$ & $1.66 \pm 0.67$ & $1.37 \pm 0.49^{\dagger}$ \\
\hline \multirow[t]{2}{*}{ Upper abdominal pain } & Placebo & $11.97 \pm 2.74$ & $9.97 \pm 2.12^{\ddagger}$ & $9.83 \pm 2.27^{\ddagger}$ \\
\hline & GCWB104 & $13.58 \pm 3.75$ & $11.26 \pm 2.70^{\ddagger}$ & $9.21 \pm 1.58^{\S}$ \\
\hline \multirow[t]{2}{*}{ Whole score } & Placebo & $22.00 \pm 4.40$ & $19.14 \pm 3.26^{\ddagger}$ & $18.66 \pm 4.30^{\ddagger}$ \\
\hline & GCWB104 & $24.21 \pm 5.26$ & $21.16 \pm 4.29^{\ddagger}$ & $18.05 \pm 2.84^{\S}$ \\
\hline
\end{tabular}

Data are presented as mean \pm SD.

GSRS, Gastrointestinal Symptom Rating Scale.

${ }^{*} \mathrm{p}<0.05,{ }^{\dagger} \mathrm{p}<0.01,{ }^{\ddagger} \mathrm{p}<0.001,{ }^{\$} \mathrm{p}<0.0001 ; \mathrm{p}$-value indicates significant changes, comparisons with baseline tested with the Wilcoxon test for matched samples; comparisons with placebo tested with the Mann-Whitney U-test. 
ables and Pearson chi-square test or Fisher exact test for categorical data. The significance of change within each treatment group was analyzed using the paired t-test or Wilcoxon signed rank test for continuity variables, and McNemar test or McNemar exact test for categorical data. Analysis of the change in
GSRS scores over the period of administration of the trial food was performed using repeated measure analysis of variance. All statistical analyses were conducted with the SAS Program version 9.4 (SAS Institute Inc, Cary, NC, USA). A one-sided test was performed for the significance test of the functional assessment,

Table 3. GSRS Score Changes after 8 Weeks of Treatment

\begin{tabular}{|c|c|c|c|}
\hline Change of GSRS & GCWB104 (n=38) & Control $(n=35)$ & p-value \\
\hline GSRS total & $-6.16 \pm 5.52^{\ddagger}$ & $-3.34 \pm 6.00^{\ddagger}$ & $0.0452^{*}$ \\
\hline GSRS total, $\%$ & $-22.85 \pm 15.62^{\ddagger}$ & $-12.20 \pm 26.16^{\dagger}$ & $0.0486^{*}$ \\
\hline Upper abdominal pain & $-4.37 \pm 3.77^{\ddagger}$ & $-2.14 \pm 3.38^{\ddagger}$ & $0.0134^{*}$ \\
\hline Abdominal pain & $-0.42 \pm 0.76^{\dagger}$ & $1.19 \pm 2.42$ & 0.1696 \\
\hline Heartburn & $-0.74 \pm 0.89^{\ddagger}$ & $-0.46 \pm 0.85^{\dagger}$ & 0.1506 \\
\hline Acid regurgitation & $-0.45 \pm 0.76^{\dagger}$ & $-0.23 \pm 0.77$ & 0.1525 \\
\hline Sensation of stomach emptiness & $-0.55 \pm 0.80^{\ddagger}$ & $-0.31 \pm 0.68^{*}$ & 0.1706 \\
\hline Nausea \& vomiting & $1.42 \pm 0.60^{\ddagger}$ & $1.31 \pm 0.53^{*}$ & 0.4524 \\
\hline Rumbling & $-0.58 \pm 0.79^{\ddagger}$ & $-0.11 \pm 0.80$ & $0.0146^{*}$ \\
\hline Abdominal distension & $-0.71 \pm 0.84^{\ddagger}$ & $-0.37 \pm 0.65^{\dagger}$ & 0.0626 \\
\hline Eructation & $-0.55 \pm 0.80^{\ddagger}$ & $-0.26 \pm 0.56^{*}$ & 0.1337 \\
\hline Increased flatus & $-0.39 \pm 0.75^{\dagger}$ & $-0.51 \pm 0.70^{\ddagger}$ & 0.4957 \\
\hline Decreased passage of stools & $-0.24 \pm 0.49^{\dagger}$ & $-0.14 \pm 0.60$ & 0.4500 \\
\hline Increased passage of stools & $-0.13 \pm 0.47$ & $-0.06 \pm 0.34$ & 0.4158 \\
\hline Loose stools & $-0.32 \pm 0.66^{*}$ & $-0.03 \pm 0.71$ & $0.0353^{*}$ \\
\hline Hard stools & $-0.05 \pm 0.70$ & $-0.34 \pm 1.24$ & 0.1109 \\
\hline Urgency for defecation & $-0.32 \pm 0.70^{*}$ & $-0.06 \pm 0.54$ & $0.0147^{*}$ \\
\hline Sensation of incomplete emptiness & $-0.34 \pm 0.71^{\dagger}$ & $-0.17 \pm 0.75$ & 0.4924 \\
\hline
\end{tabular}

Data are presented as mean \pm SD.

GSRS, Gastrointestinal Symptom Rating Scale.

${ }^{*} \mathrm{p}<0.05,{ }^{\dagger} \mathrm{p}<0.01,{ }^{\dagger} \mathrm{p}<0.001$, p-value indicates statistically significant changes; intragroup comparisons tested with the Mann-Whitney U-test; comparisons with baseline tested by the Wilcoxon test for matched samples.

Table 4. Differences between the GCWB104 and the Control Groups According to Repeated Measurements

\begin{tabular}{|c|c|c|c|c|c|c|}
\hline \multirow{2}{*}{ GSRS } & \multirow{2}{*}{ Time } & \multicolumn{3}{|c|}{ Mann-Whitney U-test } & \multicolumn{2}{|c|}{ RM-ANOVA* } \\
\hline & & Test group & Control group & p-value & $\mathrm{F}$ & $\mathrm{p}$-value \\
\hline \multirow[t]{3}{*}{ GSRS total } & 4 weeks-baseline & $-3.05 \pm 5.20$ & $-2.86 \pm 4.06$ & 0.842 & 3.769 & $0.032^{*}$ \\
\hline & 8 weeks-baseline & $-6.16 \pm 5.52$ & $-3.34 \pm 6.00$ & 0.045 & & \\
\hline & 8 weeks-4 weeks & $-3.11 \pm 3.64$ & $-0.49 \pm 4.49$ & 0.004 & & \\
\hline \multirow[t]{3}{*}{ Sensation of stomach emptiness } & 4 weeks-baseline & $-0.16 \pm 0.79$ & $-0.34 \pm 0.73$ & 0.300 & 15.011 & $0.039^{*}$ \\
\hline & 8 weeks-baseline & $-0.55 \pm 0.80$ & $-0.31 \pm 0.68$ & 0.171 & & \\
\hline & 8 weeks-4 weeks & $-0.39 \pm 0.55$ & $0.03 \pm 0.45$ & 0.001 & & \\
\hline \multirow[t]{3}{*}{ Rumbling } & 4 weeks-baseline & $-0.37 \pm 0.71$ & $-0.14 \pm 0.69$ & 0.140 & 10.105 & $0.023^{*}$ \\
\hline & 8 weeks-baseline & $-0.58 \pm 0.79$ & $-0.11 \pm 0.80$ & 0.015 & & \\
\hline & 8 weeks-4 weeks & $-0.21 \pm 0.47$ & $0.03 \pm 0.57$ & 0.061 & & \\
\hline \multirow[t]{3}{*}{ Urgency need for defecation } & 4 weeks-baseline & $-0.16 \pm 0.68$ & $0.03 \pm 0.45$ & 0.226 & 1.962 & $0.024^{*}$ \\
\hline & 8 weeks-baseline & $-0.32 \pm 0.70$ & $0.06 \pm 0.54$ & 0.015 & & \\
\hline & 8 weeks-4 weeks & $-0.16 \pm 0.44$ & $0.03 \pm 0.45$ & 0.088 & & \\
\hline
\end{tabular}

Data are presented as mean \pm SD.

GSRS, Gastrointestinal Symptom Rating Scale; RM-ANOVA, repeated measure analysis of variance.

*p<0.05; $p$-value indicates statistical significance; intragroup comparison tested by RM-ANOVA. 
and a two-sided test was performed for baseline information of subjects and safety assessment. A p-value less than 0.05 was regarded as significant.

\section{RESULTS}

\section{The state of participation and demographic characteristics}

Fig. 1 presents a flowchart of subjects progression through the study with reasons for premature discontinuation. Screening was conducted on a total of 99 subjects, and a random assignment was made to 92 of the subjects, excluding seven subjects by the selection/exclusion criteria; 46 subjects were assigned to each group (GCWB104 group, control group). Among these subjects, 91 subjects (46 in the GCWB104 group, 45 in the control group) were included in the FA group, except one who withdrew consent. During the trial, 18 people were eliminated due to violation of the selection/exclusion criteria, adverse effects, violation of the test protocol, and omission of test results, and ultimately, 73 subjects (38 in the test group, 35 in the control group) were included in the PP group. The baseline characteristics of each group are demonstrated in Table 1 . There were no significant differences in sex, age, height, or drinking habit between the two groups, and only weight showed statistically significant differences between the two groups.

\section{Validity evaluation}

\section{1) Primary efficacy evaluation}

The average GSRS score at baseline was $24.21 \pm 5.26$ in the GCWB104 group and 22.00 \pm 4.40 in the control group, with no statistically significant difference between the two groups. The improvement in GSRS score in each group was $-6.16 \pm 5.52$ in the GCWB104 group and $-3.34 \pm 6.00$ in the control group. The difference in GSRS score improvement between the two groups was statistically significant $(\mathrm{p}=0.045)$. Total GSRS scores of both groups at baseline, 4th, 8th week are shown in Table 2, and changes in total and individual symptoms of GSRS after 8 weeks of administration are shown in Table 3.

\section{2) Secondary efficacy evaluation}

(1) GSRS score improvement rate

In the PP analysis, the GSRS score improvement rate after administration of trial food compared to baseline was $-22.85 \pm 15.62$ in the trial group and $-12.20 \pm 26.16$ in the control group, and the GSRS score improvement rate between the two groups also showed a statistically significant difference $(\mathrm{p}=0.049)$.

(2) Improvement of individual symptoms of GSRS

In the analysis of improvements in individual symptoms of GSRS, a total of 13 symptoms were improved after administration of human trial food in the test group, while six were improved in the control group. As shown in Table 2, of 15 individ-

Table 5. GSRS Score Changes with 90\% Medication Compliance after 8 Weeks of Treatment

\begin{tabular}{|c|c|c|c|}
\hline Change of GSRS & GCWB104 $(n=33)$ & Control $(n=26)$ & $\mathrm{p}$-value \\
\hline GSRS total & $-6.79 \pm 5.56$ & $-3.58 \pm 4.88$ & $0.0144^{*}$ \\
\hline Abdominal pain & $-0.48 \pm 0.78$ & $-0.27 \pm 0.60$ & 0.3407 \\
\hline Heartburn & $-0.67 \pm 0.84$ & $-0.35 \pm 0.63$ & 0.1578 \\
\hline Acid regurgitation & $-0.58 \pm 0.70$ & $-0.08 \pm 0.56$ & $0.0062^{*}$ \\
\hline Sensation of stomach emptiness & $-0.61 \pm 0.78$ & $-0.23 \pm 0.59$ & $0.0464^{*}$ \\
\hline Nausea \& vomiting & $-0.42 \pm 0.60$ & $-0.31 \pm 0.55$ & 0.6271 \\
\hline Rumbling & $-0.61 \pm 0.81$ & $0.00 \pm 0.69$ & $0.0069^{*}$ \\
\hline Abdominal distension & $-0.70 \pm 0.83$ & $-0.42 \pm 0.64$ & 0.1630 \\
\hline Eructation & $-0.64 \pm 0.77$ & $-0.31 \pm 0.55$ & 0.1371 \\
\hline Increased flatus & $-0.48 \pm 0.74$ & $-0.58 \pm 0.76$ & 0.6292 \\
\hline Decreased passage of stools & $-0.27 \pm 0.51$ & $-0.23 \pm 0.59$ & 0.7401 \\
\hline Increased passage of stools & $-0.21 \pm 0.41$ & $-0.08 \pm 0.39$ & 0.2183 \\
\hline Loose stools & $-0.36 \pm 0.59$ & $-0.04 \pm 0.60$ & $0.0236^{*}$ \\
\hline Hard Stools & $-0.06 \pm 0.74$ & $-0.42 \pm 1.21$ & 0.1500 \\
\hline Urgency for defecation & $-0.33 \pm 0.72$ & $0.00 \pm 0.28$ & $0.0233^{*}$ \\
\hline Sensation of incomplete emptiness & $-0.36 \pm 0.73$ & $-0.27 \pm 0.67$ & 0.7364 \\
\hline
\end{tabular}

Data are presented as mean \pm SD.

GSRS, Gastrointestinal Symptom Rating Scale.

*p<0.05; p-value indicates statistical significance; intragroup comparisons tested by the Mann-Whitney U-test. 
ual symptoms, rumbling, loose stool, and fecal urgency showed statistically significant improvement. In these symptoms, both GSRS score improvement in the trial group before and after administration of GCWB104, and GSRS score improvement between the trial and control groups were statistically significant.

Individual symptoms can be divided into symptoms of the upper, and lower abdomen, and stomach pain, heartburn, acid reflux, hunger pain, nausea and vomiting, rumbling, bloating and eructation are symptoms of the upper abdomen that correspond to the stomach. In the statistical analysis of upper abdominal symptom GSRS score improvement, there was significant improvement in the GCWB104 group compared to the placebo group ( $\mathrm{p}=0.013)$.

(3) Improvement by compliance and duration of administration

When comparing the change from baseline to the 4th week of administration and the change from the 4th week to the end point, there was significant improvement in the GCWB104 group over time compared to the placebo group ( $\mathrm{p}=0.032$ ) (Table 4).

In the analysis of the efficacy evaluation according to compliance, a total of 59 subjects with 90\% compliance were randomly assigned to two groups, namely, GCWB104 (n=33) and placebo $(n=26)$, which were subsequently considered for analysis. After 8 weeks of treatment, GCWB104 significantly reduced the whole GSRS score compared with baseline and placebo $(p=0.014)$. Compared to placebo, administration of GCWB104 decreased symptom scores significantly in each symptom associated with upper abdominal pain, such as hunger pain, acid reflux, and rumbling. Lower abdominal symptoms, such as loose stools and urgency for defecation, were also decreased in the GCWB104 group (Table 5).

(4) Improvement of antioxidative markers

The changes in antioxidative markers are presented in Table 6. In the analysis of the changes in inflammatory and antioxidative markers, there was a statistically significant improvement in $8-0 H d G$ in the test group compared to the control group $(p=0.032)$. However, there was no significant difference in cytokine high sensitivity C-reactive protein, thiobarbituric acid reactive substances or total antioxidant levels.

(5) Improvement of dyspepsia-related quality of life

In the analysis of the improvement of dyspepsia-related quality of life using NDI, there were statistically significant changes in both trial and control groups, after 8 weeks of administration compared to baseline. However, there was no significant difference between the two groups (Table 7).

\section{Safety}

In this trial, there were 18 adverse events, seven adverse drug reactions, and 22 unexpected adverse drug reactions, in addition to four adverse events that caused cessation and one major adverse event (Table 8). However, no significant adverse reaction was associated with the test product including one major adverse event, ankle bone fracture, and no statistically significant differences in intergroup comparisons were observed.

In the analysis of vital signs, changes in systolic blood pressure within the control group and changes in pulse rates within

Table 6. Blood Biomarker Level Changes after 8 Weeks of Treatment

\begin{tabular}{lccc}
\hline Change of GSRS & GCWB104 $(\mathrm{n}=38)$ & Control $(\mathrm{n}=35)$ & $\mathrm{p}$-value \\
\hline TBARS & $0.91 \pm 5.07$ & $0.41 \pm 4.51$ & 0.2245 \\
8-OHdG & $-0.21 \pm 0.43$ & $0.01 \pm 0.43$ \\
Total antioxidant & $-0.01 \pm 0.12$ & $0.03 \pm 0.16$ \\
\hline
\end{tabular}

Data are presented as mean \pm SD.

GSRS, Gastrointestinal Symptom Rating Scale; TBARS, thiobarbituric acid reactive substances; 8-0HdG, 8-oxo-2-hydroxydeoxyguanosine. *p<0.05; $p$-value indicates statistical significance; intragroup comparisons tested with the Mann-Whitney U-test and independent two-sample ttest.

Table 7. Nepean Dyspepsia Index Changes after 8 Weeks of Treatment

\begin{tabular}{|c|c|c|}
\hline Week & GCWB104 (n=38) & Control $(n=35)$ \\
\hline Baseline & $53.55 \pm 16.93$ & $47.37 \pm 13.76$ \\
\hline 8 Weeks & $35.00 \pm 11.84$ & $32.37 \pm 7.18$ \\
\hline Change at 8 weeks from baseline & $-18.55 \pm 15.03$ & $-15.00 \pm 15.36$ \\
\hline $\mathrm{p}$-value & $0.0001^{*}$ & $0.0001^{*}$ \\
\hline p-value & & \\
\hline
\end{tabular}

Data are presented as mean \pm SD.

${ }^{*} \mathrm{p}<0.05$; $\mathrm{p}$-value indicates statistical significance; intragroup comparisons tested with the paired t-test and Wilcoxon signed rank test; intergroup comparisons tested with the Mann-Whitney U-test. 
Table 8. Adverse Events during the Study

\begin{tabular}{|c|c|c|c|}
\hline Adverse event & $\begin{array}{c}\text { GCWB104 } \\
(\mathrm{n}=46)\end{array}$ & $\begin{array}{l}\text { Control } \\
(\mathrm{n}=45)\end{array}$ & $\begin{array}{c}\text { Total } \\
(\mathrm{n}=91)\end{array}$ \\
\hline \multicolumn{4}{|l|}{ Summary of adverse experiences } \\
\hline Total adverse event & 8 & 10 & 18 \\
\hline $\mathrm{ADR}$ & 2 & 5 & 7 \\
\hline Unexpected ADR & 10 & 12 & 22 \\
\hline Adverse events that caused cessation & 2 & 2 & 4 \\
\hline Serious adverse event & 1 & 0 & 1 \\
\hline \multicolumn{4}{|l|}{ Adverse experiences } \\
\hline Eye disorders & $1(2.17)$ & 0 & $1(1.10)$ \\
\hline Cataract & $1(2.17)$ & 0 & $1(1.10)$ \\
\hline Gastrointestinal disorders & $3(6.52)$ & $3(6.67)$ & $6(6.59)$ \\
\hline Constipation & $2(4.35)^{*}$ & $0(0.00)$ & $2(2.20)$ \\
\hline Nausea & $1(2.17)$ & $1(2.22)$ & $2(2.20)$ \\
\hline Dyspepsia & 0 & $2(4.44)^{*}$ & $2(2.20)$ \\
\hline Musculoskeletal and connective tissue disorders & $3(6.52)$ & $2(4.44)$ & $5(5.49)$ \\
\hline Back pain & $1(2.17)$ & $1(2.22)$ & $2(2.20)$ \\
\hline Musculoskeletal pain & $1(2.17)$ & $1(2.22)$ & $2(2.20)$ \\
\hline Neck pain & $1(2.17)$ & 0 & $1(1.10)$ \\
\hline Respiratory, thoracic and mediastinal disorders & $1(2.17)$ & 0 & $1(1.10)$ \\
\hline Nasal obstruction & $1(2.17)$ & 0 & $1(1.10)$ \\
\hline General disorders and administration site conditions & 0 & $1(2.22)$ & $1(1.10)$ \\
\hline Edema & 0 & $1(2.22)^{*}$ & $1(1.10)$ \\
\hline Infections and infestations & 0 & $2(4.44)$ & $2(2.20)$ \\
\hline Viral upper respiratory tract infection & 0 & $2(4.44)$ & $2(2.20)$ \\
\hline Skin and subcutaneous tissue disorders & 0 & $2(4.44)$ & $2(2.20)$ \\
\hline Rash & 0 & $1(2.22)^{*}$ & $1(1.10)$ \\
\hline Urticaria & 0 & $1(2.22)^{*}$ & $1(1.10)$ \\
\hline Total patients with adverse event & $7(15.22)$ & $9(20.00)$ & $16(17.58)$ \\
\hline Total of ADR* & $2(4.35)$ & $5(11.11)$ & 7 (7.69) \\
\hline
\end{tabular}

Data are presented as number or number (\%).

GCWB, gastrointestinal symptom rating scale; $A D R$, adverse drug reactions.

*ADR reported as possibly related, probably related and definitely related by the investigator. Constipation in the test group was probably related.

the test group showed statistically significant differences but were considered to be within the normal range and have no clinical implications. In hematological and biochemical analyses, significant differences in alkaline transferase, glucose, and aspartate aminotransferase levels between the trial and control groups were observed at the time of screening. Significant differences in triglyceride and low density lipoprotein levels between the trial and control groups were observed at the 8th week of the trial, and significant differences in red blood cell, hemoglobin, hematocrit, alanine aminotransferase, and gamma glutamyl transferase were observed between the trial and control groups at the time of screening and the 8th week of the trial. Significant differences were observed in changes in albumin levels in the trial group, changes in total bilirubin levels in the control group, and changes in albumin levels between the trial and control groups. However, the changes were within the normal range and were considered to have no clinical implications.

\section{DISCUSSION}

In this study, we aimed to verify the validity and safety of GCWB104, a health functional food based on Flos Lonicera extract, in subjects with mild to moderate FD. The results showed statistically significant differences in improvement of GSRS total points over placebo, and the analysis of individual symptoms also identified improvements in rumbling, loose stool, and fecal urgency.

FD is a chronic, relapsing, and remitting disorder. The current standard for the diagnosis of FD is the Rome III criteria, which 
consist of a sensation of pain or burning in the epigastrium, early satiety, and fullness during or after a meal, or a combination of these symptoms. Treatment of FD is difficult, since it is a group of heterogeneous symptoms involving various elements of pathogenesis, and a combination of drugs that corrects the underlying pathology that can cause symptoms is needed. Treatments such as H. pylori eradication therapy, acid suppression therapy, prokinetic agents, antidepressants, and psychological therapy are available, but the overall rate of symptom resolution is only approximately 50\%. ${ }^{15}$ Approximately $15 \%$ to $20 \%$ have persistent symptoms, and the remaining 30\% to 35\% suffer fluctuations in their symptoms. In this regard, biologic drugs and health functional foods are notable. Biologic drugs and health functional foods can be superior to medicine that functions using only one mechanism, and recent studies show the effects of biologic products in FD. ${ }^{16}$ For example, DA-9701, a biological drug based on Corydalis tuber and Pharbitis seed extract, showed its effectiveness in improving gastric emptying and GI tract passage by antagonizing dopamine D2 receptors and acting on $5-\mathrm{HT}_{4}$ receptors, and it is used in the treatment of FD. ${ }^{17}$ Rikkunshito and STW-5 also showed the effect of improving GI motility and visceral pain. ${ }^{18}$ In a trial of 247 subjects, 8 weeks of Rikkunshito treatment had the effect of improving epigastric pain and postprandial satiety. ${ }^{19}$ STW- 5 has been used for about 50 years as a treatment for dyspepsia, and a study of 315 subjects with FD showed significant improvement in GI symptoms after 8 weeks of STW-5 treatment compared to placebo. $^{20,21}$

In this study, improvements in the GSRS total score and GI symptoms were identified after administration of GCWB104 for 8 weeks. A larger scale of improvement in the GSRS total score was observed in the trial group compared to the control group. In addition, in the analysis of individual symptoms of GSRS, significant improvements were observed in 13 of the 15 GSRS symptoms in the test group, while only six symptoms were improved in the control group. Of the individual symptoms, significant improvements were observed in the test group over the control group, particularly in rumbling, loose stool and fecal urgency, suggesting the ability of GCWB104 to improve GI motility in FD and irritable bowel syndrome.

The 15 individual symptoms of GSRS can be divided into upper and lower abdominal symptoms, and significant improvements in upper abdominal symptoms, including epigastric pain, heartburn, reflux, sensation of stomach emptiness, nausea and vomiting, rumbling, abdominal distention and eructation were observed in the test group compared to the control group. Since Flos Lonicera extracts have been reported to have gastric mucosal protective effects and to be effective in improving stomach function in animal experiments, ${ }^{8}$ this trial demonstrated the improvement of upper abdominal symptoms in the GCWB group, which is a meaningful result. In addition, there were also improvements in lower GI symptoms, including loose stool and fecal urgency. In a recent study of the effect of DA-9701 in a postoperative ileus animal model, DA-9701 improved GI transit and lowered plasma adrenocorticotropic hormone levels by the central corticotropin-releasing factor pathway. ${ }^{22}$ We think this can be similarly possible in GCWB104, improving GI transit by influencing the gut-brain axis. However, so far, there have been no reported research results or evidences, so further study about the pharmacologics and mechanisms of GCWB104 on the gutbrain axis in irritable bowel syndrome subjects is suggested.

It has been reported that the higher the dose compliance of the trial drug, the more effective it is, ${ }^{23-26}$ given that the drug needs a certain amount of time to act and improve symptoms in $\mathrm{FD}^{27}$ In previous clinical studies on FD, proton pump inhibitors often showed positive results in the relatively short-term period of 2 weeks, while the duration of trials with prokinetics or psychotropic drugs tended to be longer and heterogeneous, approximately 4 to 12 weeks. ${ }^{28,29}$ In this study, we set the trial period to 8 weeks, considering that GCWB104 was a health functional food with complex ingredients, and additional evaluation was performed at 4 weeks after administration to confirm the change in symptoms. In the analysis of the effects according to the duration of the administration, when a total of 8 weeks of testing was divided into 0 to 4 weeks and 4 to 8 weeks, significant improvement in symptoms could be seen over time in the test group. In addition, in the analysis of the effects according to the regimen compliance, the degree of symptom improvement in groups with a dose of 90\% was found to be significantly greater. These results are thought to be the evidence that GCWB104 improves GI symptom.

In the analysis of antioxidative biomarkers, a significant decrease in the $8-\mathrm{OHdG}$ concentration in blood in the trial group compared to the control group was found. $8-\mathrm{OHdG}$, which can be measured in blood (serum) and urine, is a representative biomarker of oxidative DNA damage. Increased levels of 8-OHdG are known to be associated with an elevated risk of DNA mutation and cancer. ${ }^{30}$ Several studies have revealed the correlation of 8-OHdG with Barrett's esophagus, adenocarcinoma of the esophagus and stomach, gastric atrophy and intestinal metaplasia. ${ }^{31,32}$ Therefore, this result is thought to be a demonstration of the antioxidative and gastric protective effect of GCWB104, because $8-0 H d G$ has been significantly decreased in the test group, although it is difficult to interpret that it contributes directly to improving GI function.

There were no safety issues with GCWB104. In this trial, there were 18 adverse events, seven adverse drug reactions, and 22 unexpected adverse drug reactions, with four adverse events that caused cessation and one major adverse event. However, no significant adverse reaction was associated with the test product, and no statistically significant differences in intergroup comparisons were observed. In the analysis of vital signs, changes in systolic blood pressure within the control group, and changes in pulse rates within the test group showed statistically 
significant differences, but were considered to be within the normal range and have no clinical implications. In hematological and biochemical analyses, there were some statistically significant changes, but all were within the normal range and were considered to have no clinical implications.

There were limitations in this study. The improvement of dyspepsia-specific quality of life after administration was observed, but there was no statistically significant difference between the trial and control groups. It is disappointing that the improvement in quality of life due to improvement of indigestion symptoms after administration of GCWB104 has not been statistically demonstrated, but since the scale of changes in NDI has been greater in the test group, it is necessary to reanalyze NDI through a larger study in the future. In addition, $H$. pylori status was analyzed by the urea breath test in the registered subjects (27 in the test group, 27 in the control group) after changing the protocol of the trial. Neither the test group nor the control group showed any significant changes. It is necessary to analyze the impact of GCWB104 on $H$. pylori, as $H$. pylori eradication is known to improve symptoms in FD. ${ }^{33}$ In this study, FD was evaluated using the translated Korean version of the validated Rome III criteria (Rome III-K questionnaire). However, Rome IV criteria were revised after confirmation of this study design, so additional studies using Rome IV criteria should be followed.

In conclusion, the results of this trial showed the potential efficacy of GCWB104 in improving GI symptoms in mild to moderate FD subjects. It also reduced some of irritable bowel symptoms, and showed antioxidant effects. So we suggest that it could be a good candidate for functional GI disorders.

\section{CONFLICTS OF INTEREST}

No potential conflict of interest relevant to this article was reported.

\section{ACKNOWLEDGEMENTS}

This work was supported by the Seoul National University Bundang Hospital Research fund (grant number: 06-2016-115). In addition, the clinical study was supported by Green Cross Wellbeing Corporation.

\section{AUTHOR CONTRIBUTIONS}

Collection, analyzation of the data and draft of the article: Y.C. Design, supervision and edition of the manuscript: N.K. Provision of the table and figures: G.T.N., J.Y.L. Study supervision: D.H.L.

\section{ORCID}

Yonghoon Choi

https://orcid.org/0000-0002-1331-969X
Nayoung Kim

https://orcid.org/0000-0002-9397-0406

Gi Tark Noh

Ju Yup Lee

Dong Ho Lee

https://orcid.org/0000-0002-3805-4835

https://orcid.org/0000-0003-0021-5354

https://orcid.org/0000-0002-6376-410X

\section{REFERENCES}

1. Knill-Jones RP. Geographical differences in the prevalence of dyspepsia. Scand J Gastroenterol Suppl 1991;182:17-24.

2. Tack J, Talley NJ, Camilleri M, et al. Functional gastroduodenal disorders. Gastroenterology 2006;130:1466-1479.

3. Kim SE, Kim N, Lee JY, et al. Prevalence and risk factors of functional dyspepsia in health check-up population: a nationwide multicenter prospective study. J Neurogastroenterol Motil 2018;24:603-613.

4. Tack J, Bisschops R, Sarnelli G. Pathophysiology and treatment of functional dyspepsia. Gastroenterology 2004;127:1239-1255.

5. Choi MG, Jung HK. Health related quality of life in functional gastrointestinal disorders in Asia. J Neurogastroenterol Motil 2011;17:245-251.

6. Oh KH, Nam Y, Jeong JH, Kim IK, Sohn UD. The effect of DA9701 on 5-hydroxytryptamine-induced contraction of feline esophageal smooth muscle cells. Molecules 2014;19:5135-5149.

7. Li Y, Cai W, Weng X, et al. Lonicerae japonicae flos and Lonicerae flos: a systematic pharmacology review. Evid Based Complement Alternat Med 2015;2015:905063.

8. Bang BW, Park D, Kwon KS, et al. BST-104, a water extract of Lonicera Japonica, has a gastroprotective effect via antioxidant and anti-inflammatory activities. J Med Food 2019;22:140-151.

9. Jung DH, Choi EJ, Jeon HH, Lee YH, Park H. Effects of GC7101, a novel prokinetic agent on gastric motor function: ex vivo study. $\mathrm{J}$ Neurogastroenterol Motil 2014;20:469-474.

10. Kang JW, Lee SM. Protective effects of chlorogenic acid against experimental reflux esophagitis in rats. Biomol Ther (Seoul) 2014;22:420-425.

11. Ku SK, Seo BI, Park JH, et al. Effect of Lonicerae flos extracts on reflux esophagitis with antioxidant activity. World J Gastroenterol 2009;15:4799-4805.

12. Rodríguez I, Illnait J, Terry H, et al. Effects of Abexol (beeswax alcohols) on gastrointestinal symptoms in middle-aged and older subjects. Revista CENIC Ciencias Biologicas 2019;40:147-154.

13. Dimenäs E, Glise H, Hallerbäck B, Hernqvist H, Svedlund J, Wiklund I. Well-being and gastrointestinal symptoms among patients referred to endoscopy owing to suspected duodenal ulcer. Scand J Gastroenterol 1995;30:1046-1052.

14. Tsai MC, Huang TL. Thiobarbituric acid reactive substances (TBARS) is a state biomarker of oxidative stress in bipolar patients in a manic phase. J Affect Disord 2015;173:22-26.

15. Talley NJ, Ford AC. Functional dyspepsia. N Engl J Med 2015;373:1853-1863.

16. Holtmann G, Talley NJ. Herbal medicines for the treatment of functional and inflammatory bowel disorders. Clin Gastroenterol 
Hepatol 2015;13:422-432.

17. Kwon YS, Son M. DA-9701: a new multi-acting drug for the treatment of functional dyspepsia. Biomol Ther (Seoul) 2013;21:181189.

18. Holtmann G, Adam B, Vinson B. Evidence-based medicine and phytotherapy for functional dyspepsia and irritable bowel syndrome: a systematic analysis of evidence for the herbal preparation Iberogast. Wien Med Wochenschr 2004;154:528-534.

19. Suzuki H, Matsuzaki J, Fukushima Y, et al. Randomized clinical trial: rikkunshito in the treatment of functional dyspepsia. A multicenter, double-blind, randomized, placebo-controlled study. Neurogastroenterol Motil 2014;26:950-961.

20. Ottillinger B, Storr M, Malfertheiner P, Allescher HD. STW 5 (Iberogast $\left.{ }^{\circledR}\right)$ : a safe and effective standard in the treatment of functional gastrointestinal disorders. Wien Med Wochenschr 2013;163:65-72.

21. von Arnim U, Peitz U, Vinson B, Gundermann KJ, Malfertheiner P. STW 5, a phytopharmacon for patients with functional dyspepsia: results of a multicenter, placebo-controlled double-blind study. Am J Gastroenterol 2007;102:1268-1275.

22. Jo SY, Hussain Z, Lee YJ, Park H. Corticotrophin-releasing factormediated effects of DA-9701 in postoperative ileus guinea pig model. Neurogastroenterol Motil 2018;30:e13385.

23. Fass R, Johnson DA, Orr WC, et al. The effect of dexlansoprazole MR on nocturnal heartburn and GERD-related sleep disturbances in patients with symptomatic GERD. Am J Gastroenterol 2011;106:421-431.

24. Lamprecht M, Bogner S, Schippinger G, et al. Probiotic supplementation affects markers of intestinal barrier, oxidation, and inflammation in trained men; a randomized, double-blinded, placebo-controlled trial. J Int Soc Sports Nutr 2012;9:45.
25. Maki KC, Reeves MS, Farmer M, et al. Green tea catechin consumption enhances exercise-induced abdominal fat loss in overweight and obese adults. J Nutr 2009;139:264-270.

26. Lambert MNT, Thorup AC, Hansen ESS, Jeppesen PB. Combined Red Clover isoflavones and probiotics potently reduce menopausal vasomotor symptoms. PLoS One 2017;12:e0176590.

27. Lee HH, Jung HK, Choi MG. Guideline recommendation for endpoints used in clinical trials for functional dyspepsia. Korean $\mathrm{J}$ Gastroenterol 2018;72:170-178.

28. Ford AC, Luthra P, Tack J, Boeckxstaens GE, Moayyedi P, Talley NJ. Efficacy of psychotropic drugs in functional dyspepsia: systematic review and meta-analysis. Gut 2017;66:411-420.

29. Yang YJ, Bang CS, Baik GH, et al. Prokinetics for the treatment of functional dyspepsia: Bayesian network meta-analysis. BMC Gastroenterol 2017;17:83.

30. Wu D, Liu B, Yin J, et al. Detection of 8-hydroxydeoxyguanosine $(8-\mathrm{OHdG})$ as a biomarker of oxidative damage in peripheral leukocyte DNA by UHPLC-MS/MS. J Chromatogr B Analyt Technol Biomed Life Sci 2017;1064:1-6.

31. Kauppi J, Räsänen J, Sihvo E, et al. Increased oxidative stress in the proximal stomach of patients with Barrett's esophagus and adenocarcinoma of the esophagus and esophagogastric junction. Transl Oncol 2016;9:336-339.

32. Albayrak F, Uyanik MH, Dursun H, et al. Should increased levels of urinary 8-hydroxydeoxyguanosine in chronic gastritis imply intestinal metaplasia or gastric atrophy? South Med J 2010;103:753-757.

33. Du LJ, Chen BR, Kim JJ, Kim S, Shen JH, Dai N. Helicobacter pylori eradication therapy for functional dyspepsia: systematic review and meta-analysis. World J Gastroenterol 2016;22:34863495. 